\title{
Boundary effects in two-band superconductors
}

\author{
Andrea Benfenati $\odot,{ }^{*}$ Albert Samoilenka $\odot$, and Egor Babaev \\ Department of Theoretical Physics, The Royal Institute of Technology, SE-10691 Stockholm, Sweden
}

(Received 27 November 2020; revised 1 April 2021; accepted 1 April 2021; published 15 April 2021)

\begin{abstract}
We present a microscopic study of the behavior of the order parameters near the boundaries of a two-band superconducting material, described by the standard tight-binding Bardeen-Cooper-Schrieffer model. We find superconducting surface states. The relative difference between bulk and surface critical temperatures is a nontrivial function of the interband coupling strength. For superconductors with weak interband coupling, boundaries induce variations of the gaps with the presence of multiple length scales, despite nonzero interband Josephson coupling.
\end{abstract}

DOI: 10.1103/PhysRevB.103.144512

\section{INTRODUCTION}

The majority of the superconductors of current interest have multiple superconducting bands [1,2] with a widely varying strength of the interband coupling. Understanding the boundary effects of a superconductor is important since superconducting currents are concentrated near the surfaces, and the physics of boundaries controls the vortex entry barriers and thus the onset of dissipation. Moreover, the behavior of the gaps near the boundaries is crucial in small superconducting devices, such as superconducting nanowires and singlephoton detectors, where multiband materials are utilized [3].

In the last decades, topological superconductors have attracted particular interest. These materials exhibit topological surface currents, the observation of which is searched as a smoking gun for topological superconductivity and can potentially be used to understand the nature of it. Among the candidate materials, there are compounds with a complicated multiband structure, raising the need for an understanding of the surface's properties [4]. For conventional and exotic multiband materials, the gaps are characterized by a variety of probes, some of which selectively probe surfaces, while others are dominated by the bulk response [5-12]. The gap ratio is a characteristic quantity that allows one to get insight into the physics of Cooper pairing. In the presence of surface superconductivity, it is therefore important to study the gap ratio properties both in the bulk and near the boundaries.

The series of experimental works [13-15] reported that, on the surface of $\mathrm{ZrB}_{12}$, the characteristics of the superconducting gaps are widely different compared to the bulk. Refs. [16,17] suggested that $\mathrm{ZrB}_{12}$ is a multiband superconductor with weak interband coupling. A partial summary of the experimentally observed discrepancies, concerning

\footnotetext{
*alben@kth.se
}

Published by the American Physical Society under the terms of the Creative Commons Attribution 4.0 International license. Further distribution of this work must maintain attribution to the author(s) and the published article's title, journal citation, and DOI. Funded by Bibsam. the surface/bulk gap structure, can be found in Table I in Ref. [14]. The surface effects are quite strong compared to other reported experimental examples of enhanced surface superconductivity [18-24]. The explain this, it was proposed to search for a mechanism of different phonon-electron interactions on the surface of the material [25]. However, recent works $[26,27]$ reported that enhanced superconductivity near the boundary is a generic property of the standard single-band Bardeen-Cooper-Schrieffer model. Namely, it was found that the presence of Friedel oscillations near the boundary induces an increase in the density of states, at a microscopic length scale, yielding a higher critical temperature [27]. The solution has multiple length scales and depends on the coherence length.

That raises the question of the nature of surface states in a generic multiband Bardeen-Cooper-Schrieffer model [1,2], where multiple length scales and energy scales are present. In this paper, we analyze the behavior of the two superconducting gaps as a function of the interband coupling. We focus on the limit of a clean ideal surface, with negligible single-particle interband scattering [28].

\section{THE MODEL}

We consider a Fermi-Hubbard Hamiltonian describing a two-band $s$-wave superconductor. For a $d$-dimensional hypercubic lattice it reads

$$
H=\sum_{i, j, \sigma, \alpha} \psi_{i \sigma \alpha}^{\dagger} h_{i j \sigma \alpha} \psi_{j \sigma \alpha}-\sum_{i \alpha, \beta} V_{\alpha \beta} \psi_{i \uparrow \alpha}^{\dagger} \psi_{i \downarrow \alpha}^{\dagger} \psi_{i \downarrow \beta} \psi_{i \uparrow \beta} .
$$

The roman indices $i, j$ label the position on a lattice with $N$ lattice points. $\sigma=\uparrow, \downarrow$ indicates the spin, while $\alpha, \beta=1,2$ label the component. Then $h_{i j \sigma \alpha}=-\mu \delta_{i j}-t \delta_{|i-j|, 1}$, where $|i-j|=1$ if $i$ and $j$ are neighboring points in a hypercubic lattice. $\mu$ is the chemical potential and $t$ the hopping coefficient. In order to ensure the Hamiltonian to be Hermitian, we have $h_{i j \sigma \alpha}=h_{j i \sigma \alpha}^{*}$ and $V_{\alpha \beta}=V_{\beta \alpha}^{*}$. Then, following the steps in Ref. [29], we perform the mean-field approximation. Introducing the Nambu spinors

$$
\begin{aligned}
& \Psi_{\alpha}^{\dagger}=\left(\psi_{1 \uparrow \alpha}^{\dagger}, \ldots, \psi_{N \uparrow \alpha}^{\dagger}, \psi_{1 \downarrow \alpha}, \ldots, \psi_{N \downarrow \alpha}\right), \\
& \Psi_{\alpha}=\left(\psi_{1 \uparrow \alpha}, \ldots, \psi_{N \uparrow \alpha}, \psi_{1 \downarrow \alpha}^{\dagger}, \ldots, \psi_{N \downarrow \alpha}^{\dagger}\right)^{T},
\end{aligned}
$$


the total mean-field Hamiltonian reads

$$
H_{\mathrm{MF}}=\sum_{\alpha=1}^{2} \Psi_{\alpha}^{\dagger} H_{\alpha} \Psi_{\alpha} .
$$

$H_{\alpha}$ is the $\alpha$-band Hamiltonian, defined as

$$
H_{\alpha}=\left(\begin{array}{cc}
h_{\uparrow \alpha} & \Delta_{\alpha} \\
\Delta_{\alpha}^{\dagger} & -h_{\downarrow \alpha}^{T}
\end{array}\right),
$$

where the elements $h_{i j \sigma \alpha}$ have been defined above. Finally, the self-consistency equations for the gaps are

$$
\Delta_{i \alpha}=\sum_{\beta=1}^{2} V_{\alpha \beta}\left\langle c_{i \uparrow \beta} c_{i \downarrow \beta}\right\rangle_{\beta} .
$$

The thermal average $\langle\cdot\rangle_{\beta}$ means it is performed over the eigenvalues of the Hamiltonian $H_{\beta}$. We can rewrite the selfconsistency equation by introducing the auxiliary vectors $\left(\mathbf{e}_{i}\right)_{j}=\delta_{i, j}$ and $\left(\mathbf{h}_{i}\right)_{j}=\delta_{j, i+N}$ as

$$
\Delta_{i \alpha}=-\sum_{\beta=1}^{2} V_{\alpha \beta} \mathbf{e}_{i} f\left(H_{\beta}\right) \mathbf{h}_{i},
$$

with $f(x)=\left(1+e^{x / T}\right)^{-1}$ being the Fermi-Dirac function. We solve self-consistently for the gaps $\Delta_{i \alpha}$, using the Chebyshev polynomial expansion method [30-32], with a polynomial up to order 1000 . The convergence criterion we adopt is $\left|\Delta_{i \alpha}^{(n+1)}-\Delta_{i \alpha}^{(n)}\right| /\left|\Delta_{i \alpha}^{(n)}\right| \leqslant 10^{-8}$, where $n$ numbers the iteration. We consider both a one-dimensional (1D) lattice with $N=1000$ sites and a 2D square lattice with $N_{x} N_{y}=60 \times 60$. The solver is a custom CUDA implementation. To calculate the critical temperatures, we solve the linearized version of the self-consistency equation (6). For details, see Ref. [26].

\section{RESULTS}

\section{A. Effects of interband coupling}

We begin by analyzing a two-band system with weak interband coupling and a similar intraband interaction in two bands. The model is rescaled so that all the quantities are given in units of the hopping coefficient $t$. We fix $\mu=0$, i.e., half-filled bands, $V_{11}=1.35$ and $V_{22}=1.36$. We compare the results for a nonzero interband interaction $V_{12}$ with the case where the bands are decoupled, i.e., $V_{12}=0.0$. In the latter, the problem is reduced to two copies of the model studied in Ref. [26] and shown to have two different critical temperatures, one for bulk states and one for boundary states. We denote by $T_{c 1}$ the bulk critical temperature, i.e., when the order parameter vanishes in the bulk. $T_{c 2}$ is the boundary critical temperature, i.e., when the order parameter vanishes on the boundaries of the superconductor. When $V_{12}=0$ the critical temperatures in the system are $T_{c 1}^{\mathrm{band} 1}=$ 0.0429 and $T_{c 2}^{\text {band } 1}=0.0536$ for band 1 , and $T_{c 1}^{\text {band } 2} \stackrel{c 1}{=} 0.045$ and $T_{c 2}^{\text {band2 }}=0.0562$ for band 2. Hence, in this example, the second band has critical temperatures $5 \%$ higher than the first. Figure 1 shows the numerically obtained gaps $\Delta_{1}$ and $\Delta_{2}$, displayed at various temperatures and interband coupling. In accordance with the results obtained by different analytical and numerical methods in Ref. [26], when $V_{12}=0$, the boundary enhancement of each gap decays to the bulk value with an independent coherence length. As the coupling is

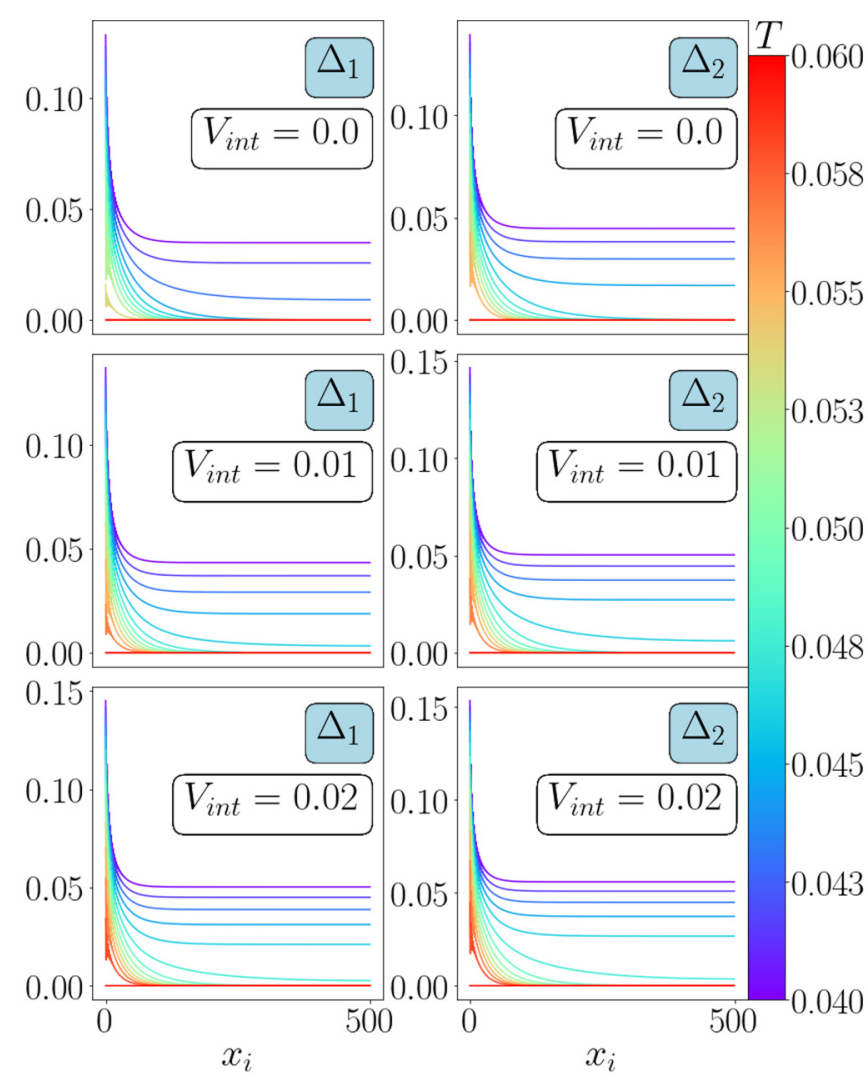

FIG. 1. Numerical solution for the two gaps at different values of the interband coupling. The boundary is located at $x=0$. For $V_{\text {int }}=0.0$, the two bands have different boundary and bulk critical temperatures. When the interband interaction is on, the two bands present the same critical temperatures. Yet, the weak interband coupling does not drastically affect the structure of the solution. The gaps exhibit different enhancements near the boundary and the overall solution shows the presence of different length scales. For $V_{12}=0.01$, we have $T_{c 1}=0.0458$ and $T_{c 2}=0.0573$. For $V_{12}=0.02$, the critical temperatures increase respectively to $T_{c 1}=0.0472$ and $T_{c 2}=0.0591$. Here, we show only the left half of the system.

turned on, $U(1)$ is broken, since the carriers in the individual bands are no longer independently conserved and there are no independent transitions for different bands. Then, for nonzero $V_{12}$, the bulk critical temperatures become the same for both bands. Also, the surface critical temperature is only one. The gap's behavior near the boundaries is nontrivial as it includes relative variations of the gap values. In the case displayed in Fig. 1 we have $T_{c 1}=0.0458$ and $T_{c 2}=0.0573$ for $V_{12}=0.01$, and $T_{c 1}=0.0472$ and $T_{c 2}=0.0591$ for $V_{12}=0.02$.

We conclude this section by moving beyond the weak interband coupling regime and investigate the relative increase of the boundary critical temperature $T_{c 2}$, with respect to the bulk temperature $T_{c 1}$, as a function of interband coupling $V_{12}$. To efficiently measure this increase, we define $\tau=$ $\left(T_{c 2}-T_{c 1}\right) / T_{c 1}$. The numerical solutions for a onedimensional model are shown in Fig. 2 for various values of $V_{22}$. We find that the dependence is nontrivial: At relatively weak interband coupling, $\tau$ first increases with $V_{12}$ and then it starts to decrease. 


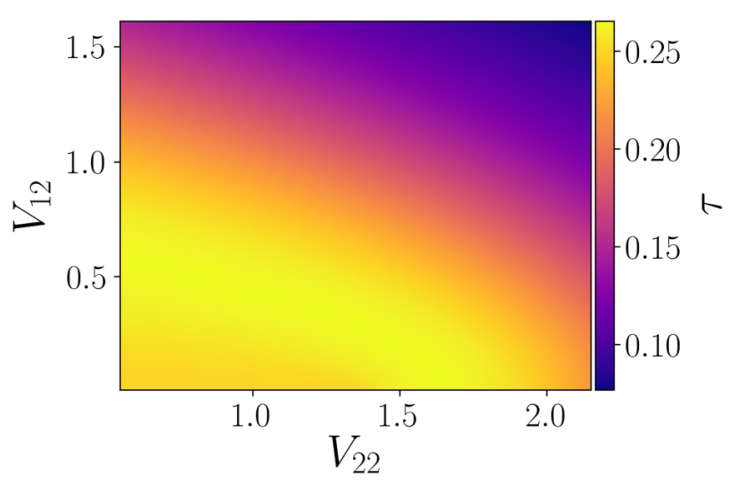

FIG. 2. Relative increase of the boundary critical temperature with respect to the bulk critical temperature as a function of the pairing potential of the second band $V_{22}$ and of the interband coupling $V_{12}$. We define $\tau=\left(T_{c 2}-T_{c 1}\right) / T_{c 1}$. For a given value of $V_{22}, \tau$ exhibits a nonmonotonic behavior as a function of interband coupling $V_{12}$. $V_{11}=1.35$ and $\mu=0$.

\section{B. The relative behavior of the gaps in two-band systems: Boundaries versus bulk}

A useful characteristic of a multiband superconductor is the ratio of the gaps of different bands, whose temperature dependence can give insights into the nature of the pairing in the material. Figure 3 shows the gap ratio shifted by its bulk values at various temperatures $T$ and interband coupling $V_{12}$ for a 1D system as a function of distance from the boundary. The system we consider first has the intraband potential of the second band only $1 \%$ bigger than the first one, namely $V_{11}=1.35$ and $V_{22}=1.36$.

Even for these similar gap characteristics, we find that the gap ratio can be different on the boundary of a superconductor compared to its bulk value, when the interband coupling is weak. Figure 3 displays the results for $V_{12}=0.01,0.02$, and compares them to the decoupled-band case.

In particular, we notice that both gaps and their ratios are enhanced near the ends of the sample, and this enhancement decays into the interior of the superconductor on a macroscopic length scale. The surface gap ratio deviation has not only a strong temperature dependence in magnitude, but also its length scale varies as a function of $T$. We can study the

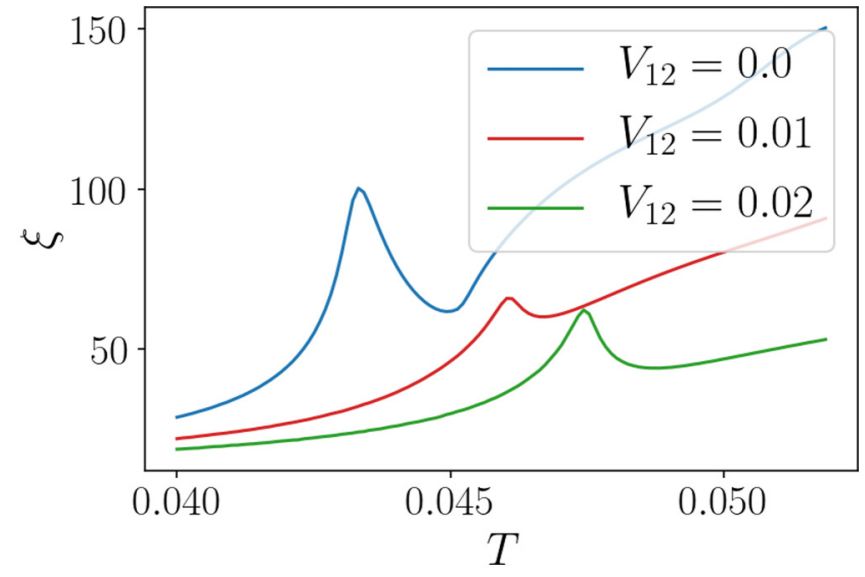

FIG. 4. Long-range asymptotic decay length scale $\xi(T)$ of the gap ratio deviation displayed in Fig. 3. $\xi(T)$ is plotted as a function of the temperature $T$ and for different values of the interband coupling. The nonmonotonic behavior as a function of $T$ is clearly visible. We obtain $\xi(T)$ by fitting the tails (i.e., after $N=50$ sites from the boundary) of the gap ratio deviation using an exponential function $f(x) \propto e^{-x / \xi}$, for the different values of $T$ and $V_{12}$. The remaining parameters used in the simulations are $V_{11}=1.35, V_{22}=1.36$, and $\mu=0$.

latter's behavior in further detail by fitting the tails (i.e., after $N=50$ sites from the boundary) of the gap ratio deviations reported in Fig. 3, with an exponential function $f(x) \propto e^{-x / \xi}$. Here, $\xi(T)$ measures the length scale of the decay into the bulk. The result, reported in Fig. 4, confirms the nonmonotonic behavior of $\xi(T)$ as a function of $T$.

Discussions concerning the existence of multiple length scales in a multiband material, previously focused on vortex physics [33-35]. The behavior of the surface states that we find is another example of the existence of multiple coherence lengths in multiband materials, despite nonzero Josephson coupling. The long-range character associated with the relative variations of the gaps and its nonmonotonicity are consistent with the conclusions obtained for the vortex core solutions in weakly interacting two-band systems in Refs. [33,34]. At higher values of the interband coupling, we can notice that the relative variation of the gap profile

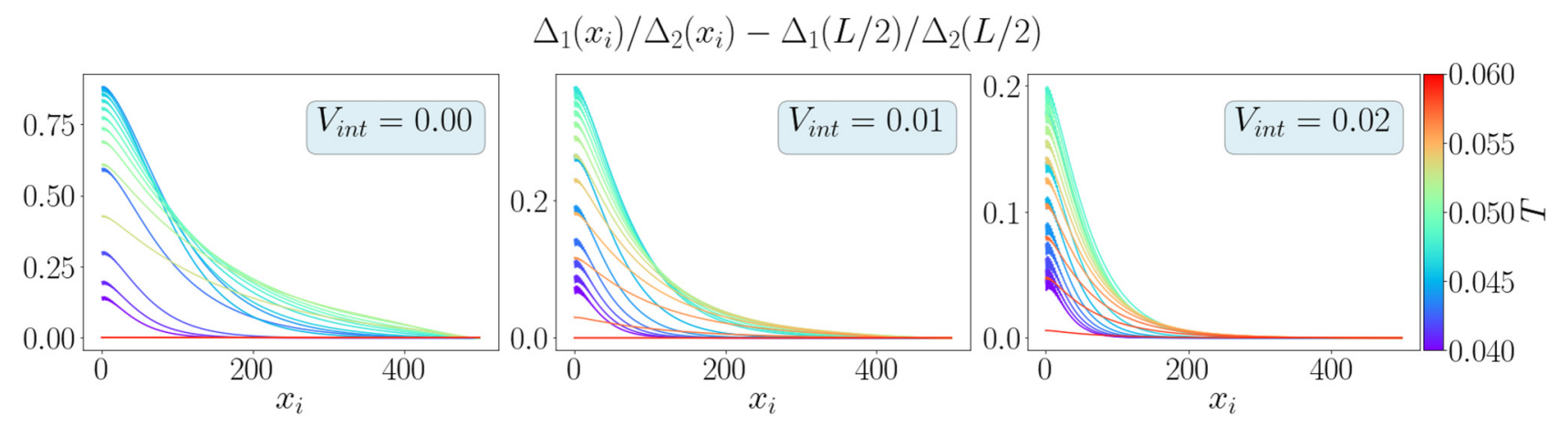

FIG. 3. Plot of gap ratios shifted by the bulk value. We can notice that the gap ratio near the surface is enhanced compared to the bulk value. The presence of weak interband coupling does not qualitatively change this effect. Moreover, the length scale of the penetration into the bulk shows a nonmonotonic behavior as a function of $T$, as more accurately displayed in Fig. 4 . We show only half of the system (500 out of $L=1000$ sites), since the second half is entirely symmetrical. The parameters used for this simulations are $V_{11}=1.35, V_{22}=1.36$, and $\mu=0$. 


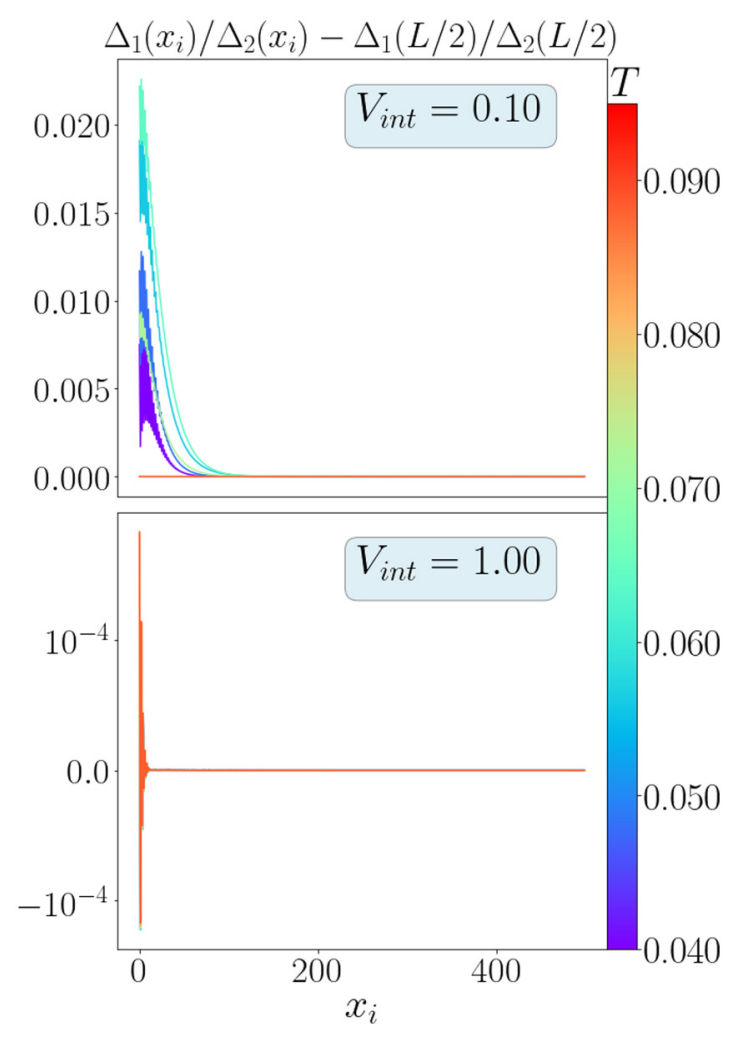

FIG. 5. Suppression of the relative gap variations at stronger interband coupling. We can notice that the gap ratio change near the boundary is an order of magnitude smaller for $V_{12}=0.1$ than for $V_{12}=0.01$. For strong interband coupling $V_{12}=1$, in the simplest two-band model, the surface-induced change of the gap ratio is negligible. Also in this case we show only half of the system (500 out of $N=1000$ sites), since the second half is symmetrical. The parameters used for these calculations are $V_{11}=1.35, V_{22}=1.36$, and $\mu=0$.

near the surfaces decreases, both in amplitude and in its spatial extension, as Fig. 5 reports. This remains consistent with the hybridization of bulk coherence lengths and their dependence on interband coupling strength [33]. When the interband coupling $V_{12}$ becomes of the same order of magnitude as the intraband coupling $V_{11}$ and $V_{22}$, e.g., $V_{12}=1.0$, the enhancement of the gap ratio basically disappears. Note that the disappearance of this variation is similar to the condition for the disappearance of the second coherence lengths in the clean two-band BCS semiclassical model found in Ref. [33]. This confirms that the width of the boundary states, in two band systems, is in general determined by two bulk coherence lengths.

Next, we consider the boundary states when the difference between the intraband potential is greater. Specifically, we consider $V_{11}=1.35$ and $V_{22}=1.68$. The upper panel of Fig. 6 shows the gaps $\Delta_{1}$ (solid line) and $\Delta_{2}$ (dashed line) at various temperatures. The bottom panel displays the variation of the gap ratio with respect to the bulk value. Here, the interband potential is set to be $V_{12}=0.1$. We can see a moderate increase of $V_{22}$ yields a substantial variation of the relative gap values near the surface compared to Fig. 5.
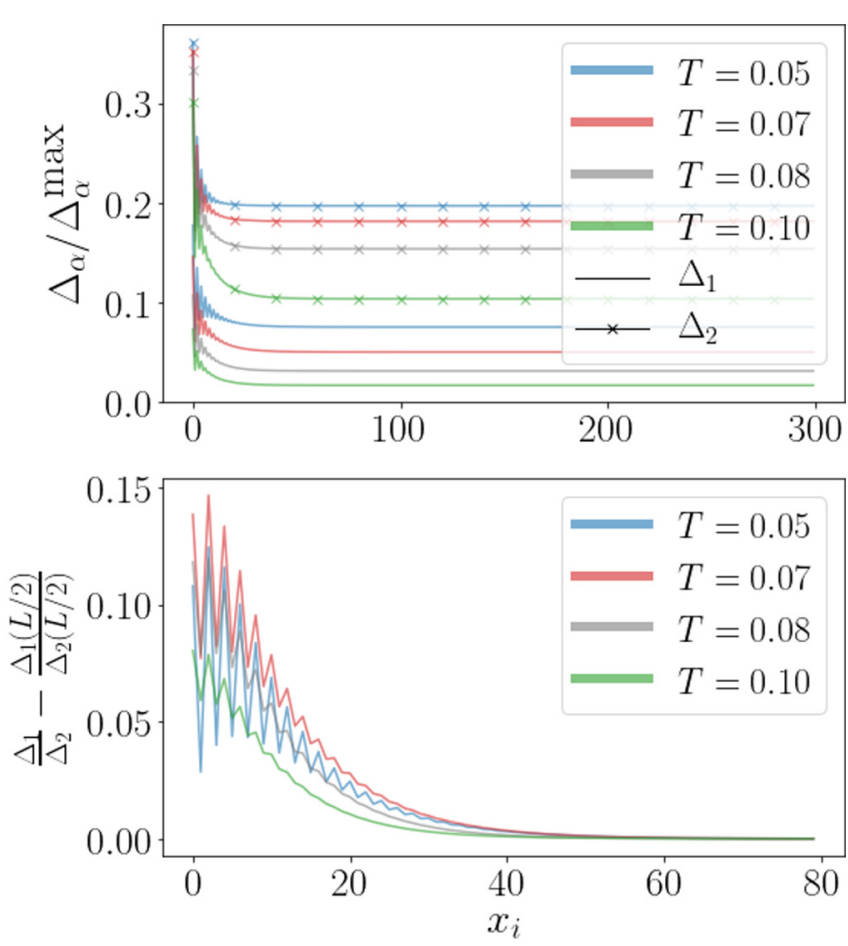

FIG. 6. Numerically obtained order parameters for different values of the $T$. In this case, we study a coupled two-band system where the difference between the two intraband potentials $V_{11}$ and $V_{22}$ is higher, namely $25 \%$. The upper panel displays the gaps $\Delta_{1}$ (solid line) and $\Delta_{2}$ (crossed line) independently. The bottom panel reports the gap ratio near-surface spatial variation relative to the bulk value. For both panels we fixed $V_{12}=0.1$. We can notice that the modest increase in $V_{22}$ results in a substantially larger variation of the gap ratio near the boundary. Both results were obtained for a system with $N=1000$ and $\mu=0$.

\section{Surface effects in two dimensions and corner states}

In $2 \mathrm{D}$ and $3 \mathrm{D}$ single-component $\mathrm{BCS}$ models there are superconducting corner and edge states with a relative critical temperature higher than the bulk critical temperature [26,29]. In this section, we consider the gap ratio spatial profile in a two-dimensional, two-band system. In 2D we have edges and corners, therefore we can associate $T_{c 2}$ as the mean-field critical temperature for edge superconductivity, and $T_{c 3}$ as the mean-field critical temperature for corner superconductivity. In a single-band BCS 2D system, $T_{c 3}>T_{c 2}$, as shown in Ref. [29]. We study the two-band system for $T<T_{c 1}, T_{c 1}<$ $T<T_{c 2}$, and $T_{c 2}<T<T_{c 3}$. Studying the boundary effects in two dimensions is challenging, as it requires numerically solving significantly large systems, to avoid the finite-size effects' influence on the resulting states. Figure 7 shows the gap ratio shifted by its value in the bulk, and the two gaps $\Delta_{1}, \Delta_{2}$. We can notice that the boundary states exist at much smaller length scales than the size of the sample. Both for the bulk $(T=0.75)$ and the edge $(T=0.77)$ states the gap ratio is enhanced along the system boundaries. When the temperature exceeds the edge critical temperature $T_{c 2}=0.774$, the superconductivity ceases to exist along the boundaries, but remains in the four corners. We observe that in the corners there is the 

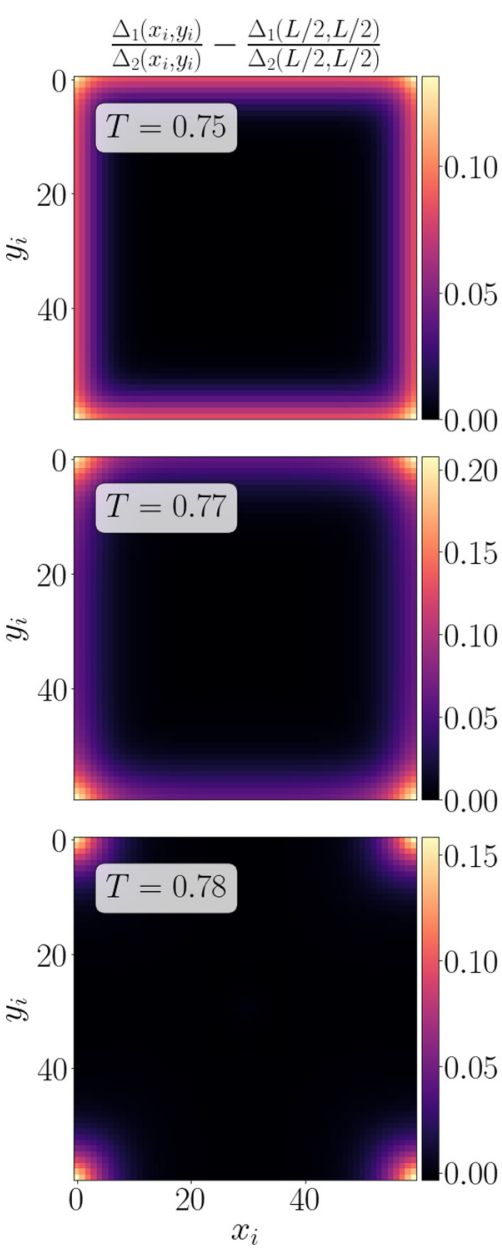
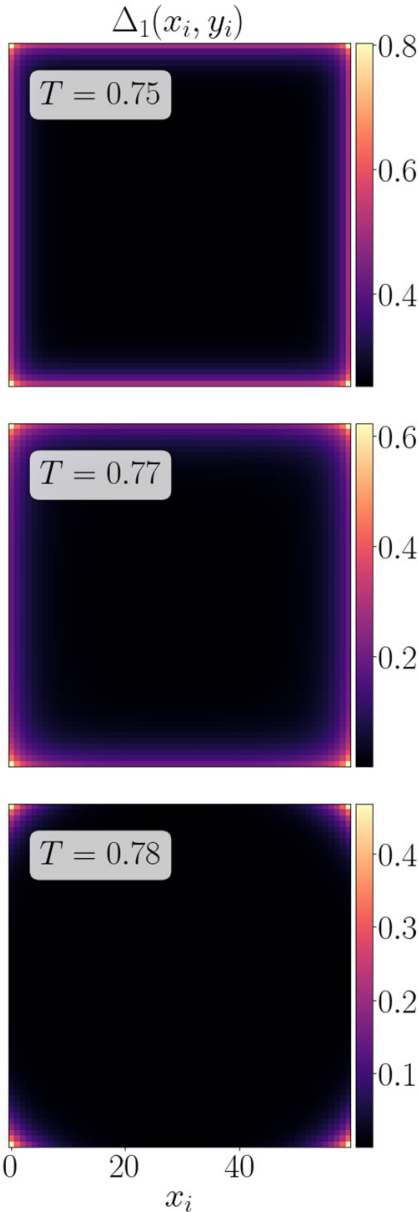
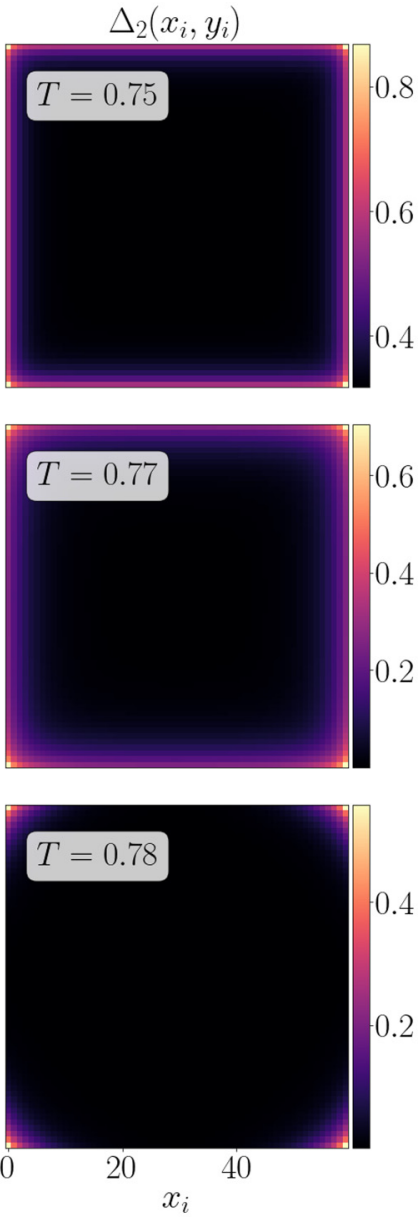

FIG. 7. Plot of the gap ratio shifted by the bulk value (left column), $\Delta_{1}$ (center column), and $\Delta_{2}$ (right column) in two dimensions for increasing temperatures. The bulk critical temperature for the system reads $T_{c 1}=0.759$ and the edge critical temperature is $T_{c 2}=0.774$. Therefore the first row shows the bulk superconductivity, the second row shows the edge superconductivity, and the third row shows the state where the gap survives only in the corners. Below the corner critical temperature, i.e., for bulk and edge states, we can notice the gradient of the gap ratio localized along the system's boundaries. The increase is more pronounced above the bulk critical temperature, i.e., with edge states. When $T_{c 2}<T<T_{c 3}$, superconductivity survives in the four corners, where also the gap ratio undergoes a significant enhancement, penetrating into the bulk with a macroscopic length scale.

largest variation of the gap ratio, which decays into the bulk at a macroscopic length scale.

\section{CONCLUSIONS}

Since most of the superconductors of current interest are multiband, and there are experimental puzzles, such as the surface gap enhancement in $\mathrm{ZrB}_{12}$ [13-15], it is important to understand the boundary effects in multiband superconducting materials.

We studied the boundary effects in the standard two-band Bardeen-Cooper-Schrieffer theory of superconductivity. We showed that, at the level of mean-field theory, the system has multiple critical temperatures, associated with the presence of boundary states.

We found that the dependence of the critical temperatures on the value of interband coupling is nonmonotonic. Moreover, when interband coupling is relatively weak, the behavior of the gaps near the boundaries presents multiple length scales and a relative variation of the gap values.
In dimensions higher than one, the effects are stronger in the sample's corners. The relative variation of the gap values extends into the superconductor with a large temperaturedependent length scale. This should be of particular importance for critical currents in small superconducting devices. The enhanced surface superconductivity may be harvested to improve superconducting-nanowire-based single-photon detectors. A material with an increased gap near the surface is expected to enhance the vortex entry barrier which can yield less dark counts. An interesting question for further studies is the effect of boundary-induced single particle scattering [36] on the states we report.

\section{ACKNOWLEDGMENTS}

The work was supported by the Swedish Research Council Grants No. 642-2013-7837, No. 2016-06122, and No. 201803659, the Göran Gustafsson Foundation for Research in Natural Sciences and Medicine, Olle Engkvists Stiftelse. 
[1] H. Suhl, B. T. Matthias, and L. R. Walker, Bardeen-CooperSchrieffer Theory of Superconductivity in the Case of Overlapping Bands, Phys. Rev. Lett. 3, 552 (1959).

[2] V. Moskalenko, Superconductivity in metals with overlapping energy bands, Fiz. Met. Metalloved. 8, 2518 (1959).

[3] H. Shibata, T. Akazaki, and Y. Tokura, Fabrication of $\mathrm{MgB}_{2}$ nanowire single-photon detector with meander structure, Appl. Phys. Express 6, 023101 (2013).

[4] A. Bouhon and M. Sigrist, Current inversion at the edges of a chiral $p$-wave superconductor, Phys. Rev. B 90, 220511(R) (2014).

[5] A. Kreisel, P. J. Hirschfeld, and B. M. Andersen, On the remarkable superconductivity of FeSe and its close cousins, Symmetry 12, 1402 (2020).

[6] A. Chubukov, Pairing mechanism in Fe-based superconductors, Annu. Rev. Condens. Matter Phys. 3, 57 (2012).

[7] I. Mazin and V. Antropov, Electronic structure, electronphonon coupling, and multiband effects in $\mathrm{MgB}_{2}$, Physica C: Superconductivity 385, 49 (2003).

[8] A. P. Mackenzie, T. Scaffidi, C. W. Hicks, and Y. Maeno, Even odder after twenty-three years: The superconducting order parameter puzzle of $\mathrm{Sr}_{2} \mathrm{RuO}_{4}$, npj Quantum Mater. 2, 40 (2017).

[9] R. Sharma, S. D. Edkins, Z. Wang, A. Kostin, C. Sow, Y. Maeno, A. P. Mackenzie, J. S. Davis, and V. Madhavan, Momentum-resolved superconducting energy gaps of $\mathrm{Sr}_{2} \mathrm{RuO}_{4}$ from quasiparticle interference imaging, Proc. Natl. Acad. Sci. USA 117, 5222 (2020).

[10] J. Böker, P. A. Volkov, K. B. Efetov, and I. Eremin, $s+i s$ superconductivity with incipient bands: Doping dependence and STM signatures, Phys. Rev. B 96, 014517 (2017).

[11] S. Z. Zhao, H.-Y. Song, L. L. Hu, T. Xie, C. Liu, H. Q. Luo, C.-Y. Jiang, X. Zhang, X. C. Nie, J.-Q. Meng, Y. X. Duan, S. B. Liu, H. Y. Xie, and H. Y. Liu, Observation of soft Leggett mode in superconducting $\mathrm{CaKFe}_{4} \mathrm{As}_{4}$, Phys. Rev. B 102, 144519 (2020).

[12] G. Rubio-Bollinger, H. Suderow, and S. Vieira, Tunneling Spectroscopy in Small Grains of Superconducting $\mathrm{MgB}_{2}$, Phys. Rev. Lett. 86, 5582 (2001).

[13] M. I. Tsindlekht, G. I. Leviev, I. Asulin, A. Sharoni, O. Millo, I. Felner, Y. B. Paderno, V. B. Filippov, and M. A. Belogolovskii, Tunneling and magnetic characteristics of superconducting $\mathrm{ZrB}_{12}$ single crystals, Phys. Rev. B 69, 212508 (2004).

[14] M. Belogolovskii, I. Felner, and V. Shaternik, Zirconium dodecaboride, a novel superconducting material with enhanced surface characteristics, Boron Rich Solids (Springer, Berlin, 2010), pp. 195-206.

[15] R. Khasanov, D. Di Castro, M. Belogolovskii, Y. Paderno, V. Filippov, R. Brütsch, and H. Keller, Anomalous electronphonon coupling probed on the surface of superconductor $\mathrm{ZrB}_{12}$, Phys. Rev. B 72, 224509 (2005).

[16] V. A. Gasparov, N. S. Sidorov, and I. I. Zver'kova, Two-gap superconductivity in $\mathrm{ZrB}_{12}$ : Temperature dependence of critical magnetic fields in single crystals, Phys. Rev. B 73, 094510 (2006).

[17] P. K. Biswas, F. N. Rybakov, R. P. Singh, S. Mukherjee, N. Parzyk, G. Balakrishnan, M. R. Lees, C. D. Dewhurst, E. Babaev, A. D. Hillier, and D. M. Paul, Coexistence of type-I and type-II superconductivity signatures in $\mathrm{ZrB}_{12}$ probed by muon spin rotation measurements, Phys. Rev. B 102, 144523 (2020).
[18] H. Fink and W. Joiner, Surface Nucleation and Boundary Conditions in Superconductors, Phys. Rev. Lett. 23, 120 (1969).

[19] R. Lortz, T. Tomita, Y. Wang, A. Junod, J. Schilling, T. Masui, and $\mathrm{S}$. Tajima, On the origin of the double superconducting transition in overdoped $\mathrm{YBa}_{2} \mathrm{Cu}_{3} \mathrm{O}_{x}$, Physica C: Superconductivity 434, 194 (2006).

[20] E. Janod, A. Junod, T. Graf, K.-Q. Wang, G. Triscone, and J. Muller, Split superconducting transitions in the specific heat and magnetic susceptibility of $\mathrm{YBa}_{2} \mathrm{Cu}_{3} \mathrm{O}_{x}$ versus oxygen content, Physica C: Superconductivity 216, 129 (1993).

[21] R. A. Butera, High-resolution heat capacity of $\mathrm{YBa}_{2} \mathrm{Cu}_{3} \mathrm{O}_{6.9}$ over the superconducting transition region, Phys. Rev. B 37, 5909 (1988).

[22] I. Khlyustikov, Critical magnetic field of surface superconductivity in lead, J. Exp. Theor. Phys. 113, 1032 (2011).

[23] I. Khlyustikov, Surface superconductivity in lead, J. Exp. Theor. Phys. 122, 328 (2016).

[24] I. Mangel, I. Kapon, N. Blau, K. Golubkov, N. Gavish, and A. Keren, Stiffnessometer: A magnetic-field-free superconducting stiffness meter and its application, Phys. Rev. B 102, 024502 (2020).

[25] V. Ginzburg, On surface superconductivity, Phys. Lett. 13, 101 (1964).

[26] A. Samoilenka and E. Babaev, Boundary states with elevated critical temperatures in Bardeen-Cooper-Schrieffer superconductors, Phys. Rev. B 101, 134512 (2020).

[27] A. Samoilenka and E. Babaev, Microscopic derivation of superconductor-insulator boundary conditions for GinzburgLandau theory revisited. Enhanced superconductivity with and without magnetic field, arXiv:2011.09519.

[28] The effects of single-particle interband scattering were already studied in Ref. [36]. The effects of atomic-scale surface imperfections were addressed in Ref. [26] for a single-band material.

[29] A. Samoilenka, M. Barkman, A. Benfenati, and E. Babaev, Pairdensity-wave superconductivity of faces, edges, and vertices in systems with imbalanced fermions, Phys. Rev. B 101, 054506 (2020).

[30] A. Weiße, G. Wellein, A. Alvermann, and H. Fehske, The kernel polynomial method, Rev. Mod. Phys. 78, 275 (2006).

[31] L. Covaci, F. M. Peeters, and M. Berciu, Efficient Numerical Approach to Inhomogeneous Superconductivity: The Chebyshev-Bogoliubov-de Gennes Method, Phys. Rev. Lett. 105, 167006 (2010).

[32] Y. Nagai, Y. Ota, and M. Machida, Efficient numerical self-consistent mean-field approach for fermionic many-body systems by polynomial expansion on spectral density, J. Phys. Soc. Jpn. 81, 024710 (2012).

[33] M. Silaev and E. Babaev, Microscopic theory of type-1.5 superconductivity in multiband systems, Phys. Rev. B 84, 094515 (2011).

[34] M. Silaev and E. Babaev, Microscopic derivation of twocomponent Ginzburg-Landau model and conditions of its applicability in two-band systems, Phys. Rev. B 85, 134514 (2012).

[35] J. Carlström, E. Babaev, and M. Speight, Type-1.5 superconductivity in multiband systems: Effects of interband couplings, Phys. Rev. B 83, 174509 (2011).

[36] E. Bascones and F. Guinea, Surface effects in two-band superconductors: Application to $\mathrm{MgB}_{2}$, Phys. Rev. B 64, 214508 (2001). 